\title{
Integration and Adoption Analysis of Digital Health Monitoring Devices: Reflections of a Pilot Project
}

Christopher Park, BA; Emmamuzo Otobo, MD, MPH; Jason Rogers, BA; Farah Fasihuddin, MPH; Shashank Garg, MS; Sarthak Kakkar, MS; Sean Pinney, MD; Jennifer Ullman, NP; Chloe Yang, BA; Zahin Roja; Kritika Singh; Vinod Kumar; Divya Madisetty, MD; Ashish Atreja, MD, MPH

Icahn School of Medicine at Mount Sinai, New York, NY, United States

Corresponding Author:

Christopher Park, BA

Icahn School of Medicine at Mount Sinai

1 Gustave L Levy Place

New York, NY,

United States

Phone: 2416500

Email: chris.park@icahn.mssm.edu

\section{Abstract}

Background: Congestive heart failure (CHF) is a disease that affects about 6.5 million people in the United States with a mortality rate of around $30 \%$. With the incidence rate projected to rise by $46 \%$ to exceed 8 million cases by 2030 , projections estimate that total CHF costs will increase about to nearly $\$ 70$ billion. Recently, the advent of remote monitoring technology has significantly broadened the scope of the physician's reach in chronic disease management. Using remotely monitored health data, providers may be able to better manage and predict their patients' outcomes, leading to reduced incidence and hospital admission costs.

Objective: This project aimed to demonstrate the feasibility of a digital medicine engagement platform for CHF patients, including identifying factors associated with increased risk of readmission and assessing usage patterns of remote monitoring devices.

Methods: The project included 60 patients admitted to Mount Sinai Hospital for CHF. A digital medicine platform by Rx.Health, called RxUniverse, was used to prescribe HealthPROMISE and iHealth mobile apps. Patients updated and recorded their CHF-related symptoms and quality of life measures daily on HealthPROMISE. Vital sign data, including blood pressure and weight, were collected through an ambulatory remote monitoring system that integrated the iHealth app and complementary consumer grade Bluetooth-connected smart devices (blood pressure cuff and digital scale). Physicians were notified of abnormal patient blood pressure and weight change readings and further action was left to the physician's discretion. We used statistical analyses to determine risk factors associated with 30-day all-cause readmission.

Results: Overall, there were six 30-day hospital readmissions (10\%), compared to the national readmission rates of around $25 \%$. Single marital status $(P<.1)$ and history of percutaneous coronary intervention $(P<.1)$ were associated with readmission. Readmitted patients were also less likely to have been previously prescribed angiotensin converting enzyme inhibitors or angiotensin II receptor blockers $(P<.05)$. Notably, readmitted patients utilized the blood pressure and weight monitors less than non-readmitted patients, and patients aged less than 70 used the monitors more frequently on average than those over 70, though these trends did not reach statistical significance. The percentage of patients using the monitors at least once dropped steadily from $83 \%$ in the first week after discharge to $46 \%$ in the fourth week. Additionally, $88 \%$ of patients used the monitor at least 4 times and $62 \%$ at least 10 times, with some patients using the monitors multiple times per day.

Conclusions: Given the increasing burden of CHF, there is a need for an effective and sustainable remote monitoring system for CHF patients following hospital discharge. We identified clinical and social factors as well as remote monitor usage trends that identify targetable patient populations that could benefit most from integration of daily remote monitoring. In addition, we demonstrated that interventions driven by real-time vitals data may greatly aid in reducing hospital readmissions and costs while improving patient outcomes. Future studies should seek to implement remote monitoring and confirm usage trends as well as risk factors in a large-scale population.

(iproc 2018;4(2):e11810) doi: $10.2196 / 11810$ 


\section{KEYWORDS}

congestive heart failure; innovation adoption process; mobile health intervention; remote monitoring

Edited by T Hale; this is a non-peer-reviewed article. Submitted 02.08.18; accepted 29.08.18; published 17.09.18.

Please cite as:

Park C, Otobo E, Rogers J, Fasihuddin F, Garg S, Kakkar S, Pinney S, Ullman J, Yang C, Roja Z, Singh K, Kumar V, Madisetty D, Atreja A

Integration and Adoption Analysis of Digital Health Monitoring Devices: Reflections of a Pilot Project iproc 2018;4(2):e11810

URL: http://www.iproc.org/2018/2/e11810/

doi: $\underline{10.2196 / 11810}$

PMID:

(C) Christopher Park, Emmamuzo Otobo, Jason Rogers, Farah Fasihuddin, Shashank Garg, Sarthak Kakkar, Sean Pinney, Jennifer Ullman, Chloe Yang, Zahin Roja, Kritika Singh, Vinod Kumar, Divya Madisetty, Ashish Atreja. Originally published in Iproceedings (http://www.iproc.org), 17.09.2018. This is an open-access article distributed under the terms of the Creative Commons Attribution License (https://creativecommons.org/licenses/by/4.0/), which permits unrestricted use, distribution, and reproduction in any medium, provided the original work, first published in Iproceedings, is properly cited. The complete bibliographic information, a link to the original publication on http://www.iproc.org/, as well as this copyright and license information must be included. 